\title{
Histopathologic spectrum and immunohistochemical diagnosis of amebic meningoencephalitis
}

\author{
Jeannette Guarner ${ }^{1}$, Jeanine Bartlett ${ }^{1}$, Wun-Ju Shieh ${ }^{1}$, Christopher D Paddock ${ }^{1}$, \\ Govinda S Visvesvara ${ }^{2}$ and Sherif R Zaki ${ }^{1}$ \\ ${ }^{1}$ Infectious Disease Pathology Branch, National Center for Infectious Diseases, Centers for Disease Control
and Prevention, Atlanta, GA, USA and ${ }^{2}$ Division of Parasitic Diseases, National Center for Infectious
Diseases, Centers for Disease Control and Prevention, Atlanta, GA, USA
}

Traditionally, Naegleria fowleri infections are labeled primary amebic meningoencephalitis because of prominent meningeal neutrophilic inflammation. Acanthamoeba spp. and Balamuthia mandrillaris are labeled granulomatous amebic encephalitis because of parenchymal granulomatous inflammation. We compared histopathologic and immunohistochemical features of 18 cases with central nervous system free-living ameba infections. Immunohistochemical assays using polyclonal antibodies that reacted specifically against each genus identified 11 patients with Balamuthia infection, four with $\mathbf{N}$. fowleri, and three with Acanthamoeba. Immunohistochemical assays highlighted the presence of trophozoites that were difficult to identify with hematoxylin and eosin stains in areas of necrosis or where macrophages were abundant. Immunohistochemical assays also demonstrated the presence of granular antigens inside macrophages and blood vessel walls. Amebic cysts were observed in three patients with Acanthamoeba infection and in three with Balamuthia. Patients with Acanthamoeba infection showed granulomatous inflammation. Patients with Naegleria infection had neutrophilic inflammation. Balamuthia infections showed a spectrum of inflammation that ranged from primarily neutrophils to granulomas. Meningitis was present in $88 \%$ of cases. Immunohistochemical assays were useful to demonstrate the presence of granular antigens and confirmed the genus of the ameba. The spectrum of inflammation in cases of Balamuthia meningoencephalitis is broader than previously described. The term amebic meningoencephalitis describes better the histopathologic findings than the currently used classification of primary amebic meningoencephalitis and granulomatous amebic encephalitis.

Modern Pathology (2007) 20, 1230-1237; doi:10.1038/modpathol.3800973; published online 12 October 2007

Keywords: immunohistochemistry; meningoencephalitis; free-living ameba; Naegleria fowleri; Balamuthia sp.; Acanthamoeba spp.

Acanthamoeba, Balamuthia, and Naegleria are three genera of free-living amebas that contain species that are pathogenic to humans. Acanthamoeba spp. and Balamuthia mandrillaris cause subacute or chronic infections of the central nervous system known as granulomatous amebic encephalitis. ${ }^{1-4}$ The histopathologic process is characterized by parenchymal necrosis and granulomas, with variations in the amount of inflammation that are dependent on the immunologic status of the host. Acanthamoeba and Balamuthia trophozoites and cysts are usually found in and around the wall of

Correspondence. Current address: Dr J Guarner, MD, Department of Pathology and Laboratory Medicine, Emory University School of Medicine, 1405 Clifton Road, NE, Atlanta, GA 30322, USA.

E-mail: Jeanette.Guarner@choa.org

Received 26 June 2007; revised 24 August 2007; accepted 5 September 2007; published online 12 October 2007 small to medium-sized blood vessels causing an angiitis. This angiitis is usually accompanied by hemorrhage, thrombosis, and necrosis. In contrast, the rapidly fatal infection caused by Naegleria fowleri causes prominent meningeal inflammation and is known as primary amebic meningoencephalitis. The histopathologic process in cases with primary amebic meningoencephalitis is characterized by a fibrinopurulent leptomeningitis associated with necrosis, hemorrhage, and edema of the brain parenchyma. N. fowleri trophozoites are usually observed in the perivascular spaces and cysts are usually absent. ${ }^{1-4}$

Serologic assays have been used for premortem diagnosis of amebic infections with inconsistent success. ${ }^{5,6}$ Other diagnostic techniques, including microscopy of wet mounts, histopathology, electron microscopy, immunocytochemistry (such as immunofluorescence, flow cytometry), and culture, rely 
on identification of trophozoites and cysts in cerebrospinal fluid or brain tissue specimens. ${ }^{1,7,8}$ Previous histopathologic reports describe single cases or small series of patients infected by one amebic genus. ${ }^{9-20}$ Side-by-side histopathologic comparison of cases caused by different ameba is not available. To better characterize the central nervous system pathology caused by the three different genera of free-living ameba, we evaluated the histopathologic and immunohistochemical features of patients infected with Acanthamoeba, Balamuthia, and Naegleria.

\section{Materials and Methods}

Immunohistochemical assays for free-living amebas were applied to formalin-fixed, paraffin-embedded tissue specimens from 42 patients with clinically or pathologically suspected amebic central nervous system infections. These samples were sent for consultation to the Centers for Disease Control and Prevention (CDC; Atlanta, GA, USA) from 1998 to 2006. Eighteen patients were included in the study based on morphologic and immunohistochemical evidence of free-living amebic meningoencephalitis. Demographic and clinical data varied in completeness. Hematoxylin and eosin stains were evaluated for the type of inflammation and angiitis as defined in Table 1, and presence of necrosis, hemorrhage, trophozoites, and cysts in the meninges and brain parenchyma.

\section{Immunohistochemistry}

Immunohistochemical assays were performed as previously described for other infectious agents. ${ }^{21,22}$ Sections $(3 \mu \mathrm{m})$ of the tissues were placed on

Table 1 Histopathologic description of angiitis and type of inflammation used in the cases

\begin{tabular}{ll}
\hline Feature & Description \\
\hline Angiitis & $\begin{array}{l}\text { Infiltration and damage of a vessel wall } \\
\text { by inflammatory cells and protozoa }\end{array}$ \\
Acute inflammation & $\begin{array}{l}\text { Neutrophils were the most prominent } \\
\text { inflammatory cell component present }\end{array}$ \\
Chronic inflammation & $\begin{array}{l}\text { Lymphocytes and macrophages were the } \\
\text { predominant inflammatory component }\end{array}$ \\
Mixed inflammation & $\begin{array}{l}\text { Equal proportion of neutrophils, } \\
\text { lymphocytes, and macrophages was } \\
\text { present }\end{array}$ \\
Granulomatous & $\begin{array}{l}\text { Presence of foamy macrophages and } \\
\text { multinucleated giant cells accompanied } \\
\text { inflammation }\end{array}$ \\
by lymphocytes \\
$\begin{array}{l}\text { Mixed granulomatous } \\
\text { inflammation }\end{array}$ & $\begin{array}{l}\text { Presence of foamy macrophages and } \\
\text { multinucleated giant cells accompanied } \\
\text { by neutrophils }\end{array}$
\end{tabular}

Colormark Plus slides (Erie Scientific Co., Portsmouth, NH, USA), deparaffinized, and rehydrated through graded alcohols and distilled water. Tissue sections were washed in Tris-saline-tween 20 buffer ( $\mathrm{pH}$ 7.5) and placed in a DAKO Autostainer (Dako, Carpinteria, CA, USA). Digestion was performed by soaking slides in $0.1 \mathrm{mg} / \mathrm{ml}$ proteinase $\mathrm{K}$ (Roche, Indianapolis, IN, USA) in 0.6 Tris-salinetween $/ 0.1 \% \mathrm{CaCl}_{2}$ (buffer) for $15 \mathrm{~min}$ at room temperature, followed by a blocking step with $20 \%$ normal sheep serum in Tris-saline-tween. Tissue sections were incubated for $60 \mathrm{~min}$ with either polyclonal rabbit anti-Acanthamoeba castellanii, Balamuthia mandrillaris, or Naegleria fowleri antibodies, respectively (Parasitic Diseases Branch, CDC). Optimal dilutions of the antibodies had been determined in previous experiments using formalinfixed cultures of the three genera of ameba (1:30 000 for the Acanthamoeba and Balamuthia antibodies and 1:20000 for the Naegleria antibody). After being incubated with the primary antibody, slides were washed, and swine anti-rabbit biotinylated link antibody, alkaline phosphatase-labeled streptavidin, and naphthol fast red chromogenic substrate (LSAB2 Universal alkaline phosphatase system, Dako) were sequentially applied. Sections were then counterstained in Mayer's hematoxylin (Fisher Scientific, Pittsburgh, PA, USA).

Each polyclonal antibody reacted against formalin-fixed, paraffin-embedded cultures of the specific ameba genus it was raised against (for example, the anti-Acanthamoeba antibody reacted against $A$. culbertsoni and $A$. castellanii) and did not crossreact with formalin-fixed cultures of the other genera of free-living ameba that were tested. Negative tissue controls consisted of sequential sections incubated with the other anti-ameba antibodies. Interpretation of immunohistochemical results included evaluation of: (1) the location of the positive reaction (ie, necrotic brain parenchyma, meninges, or blood vessels), (2) the parasite staining pattern (trophozoites, cysts, or granular antigen staining), and (3) the strength of the reaction compared to the tissue control.

\section{Results}

Table 2 presents age, sex, duration of illness, and pathologic features of the 18 patients with amebic meningoencephalitis. All patients expired except for two. The first was a 33-year-old male receiving highly active antiretroviral treatment for AIDS with CD4 cell counts of $82 / \mathrm{mm}^{3}$ who had a discrete brain lesion that showed a granulomatous Acanthamoeba infection on a biopsy specimen; he survived 22 months after having been treated with fluoconazole and pyrimethamine. ${ }^{13}$ The second patient was a 5 -year-old female who presented with seizures that were attributed to two distinct lesions in the temporal and parietal lobes; Balamuthia infection 


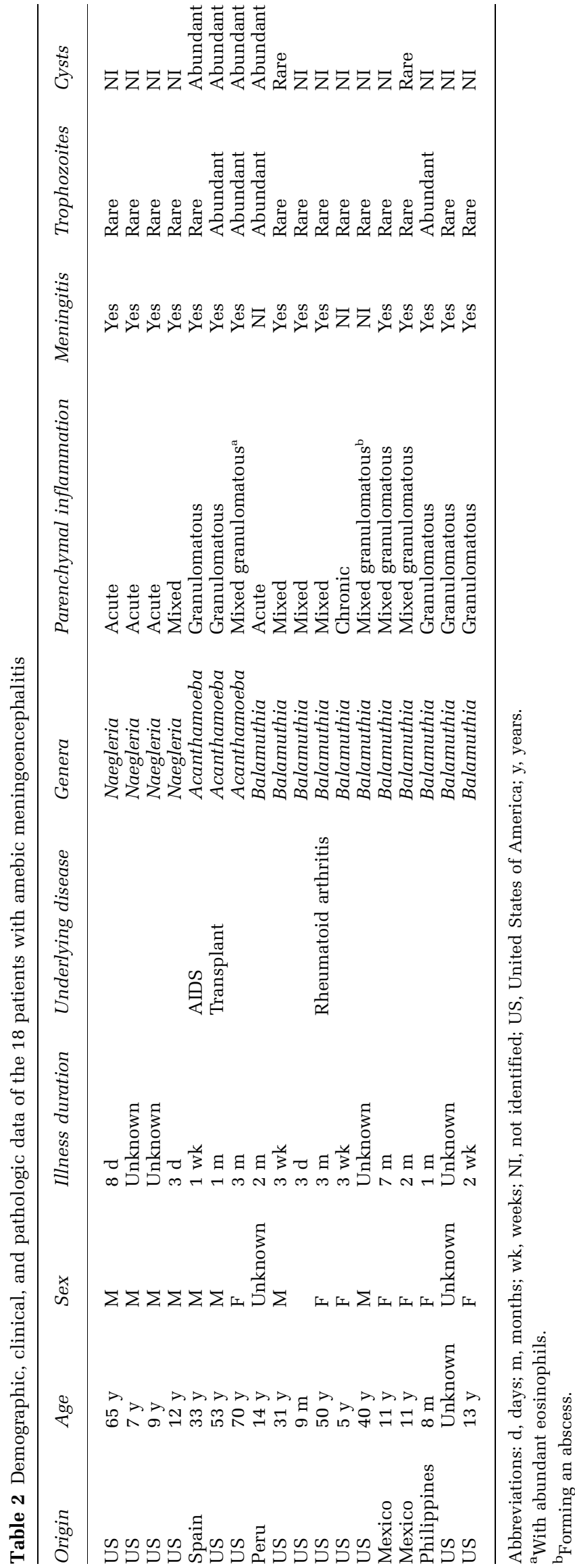

was diagnosed in a biopsy sample, and she was alive 2 years after having received a variety of treatments including ketoconazole, flucytosine, clarithromycin, and pentamidine. ${ }^{19}$

Information regarding the presence of an underlying health problem was available for three patients. A 53-year-old woman who had been treated with a bone marrow transplant for chronic lymphocytic leukemia, a 50-year-old woman with rheumatoid arthritis, and the 33-year-old man with AIDS already described. ${ }^{13}$ Information about duration of illness was incomplete but the patients with $N$. fowleri generally died within days following symptom onset, while the duration of symptoms was much broader (ranging from days to months) for patients with Acanthamoeba and Balamuthia infections.

Parasite morphology and immunohistochemical assays demonstrated the presence of Balamuthia in 11 patients, Naegleria in four, and Acanthamoeba in three patients. All cases showed necrotizing encephalitis (Figure 1a), 14 had angiitis (Figure 1b), and 10 had hemorrhage. The type of inflammation present in the brain parenchyma of each case is presented in Table 2. Patients with Naegleria showed predominantly acute inflammation (Figure 1a), Acanthamoeba infected patients mostly had granulomatous inflammation (Figure 1c), and those with Balamuthia showed a spectrum from acute (Figure 1d) to granulomatous inflammation. Eosinophils were prominent in only one patient with Acanthamoeba infection.

Ameba trophozoites were usually observed around vessels in the brain parenchyma of all cases; however, in most cases, trophozoites proved to be difficult to identify on initial review of hematoxylin and eosin stains because of intense infiltrates by macrophages or presence of necrotic debris. The trophozoites were readily evident with hematoxylin and eosin stains in two patients with Acanthamoeba (Figure 2a) infection and two with Balamuthia (Figure 2b). Amebic cysts were observed in the three patients with Acanthamoeba (Figure 2a) infection and in three with Balamuthia (Figure 2b).

Meninges were available for study in 17 cases and inflammation was present in 15 (88\%). There was acute meningeal inflammation in the four cases with Naegleria infection. Two patients with Acanthamoe$b a$ showed mixed inflammation and one chronic inflammation in the meninges. Patients with Balamuthia infection showed a spectrum of inflammatory meningeal infiltrates including two with mixed inflammation, four with chronic, one with mixed granulomatous and one with granulomatous. Five $(28 \%)$ cases showed trophozoites in the meninges, two had Naegleria, two Balamuthia, and one Acanthamoeba. None of the cases showed cysts in the meninges.

Trophozoites of each species stained strongly with the specific immunohistochemical assay (Figure 3a and $b$ ) including those present in areas of necrosis 

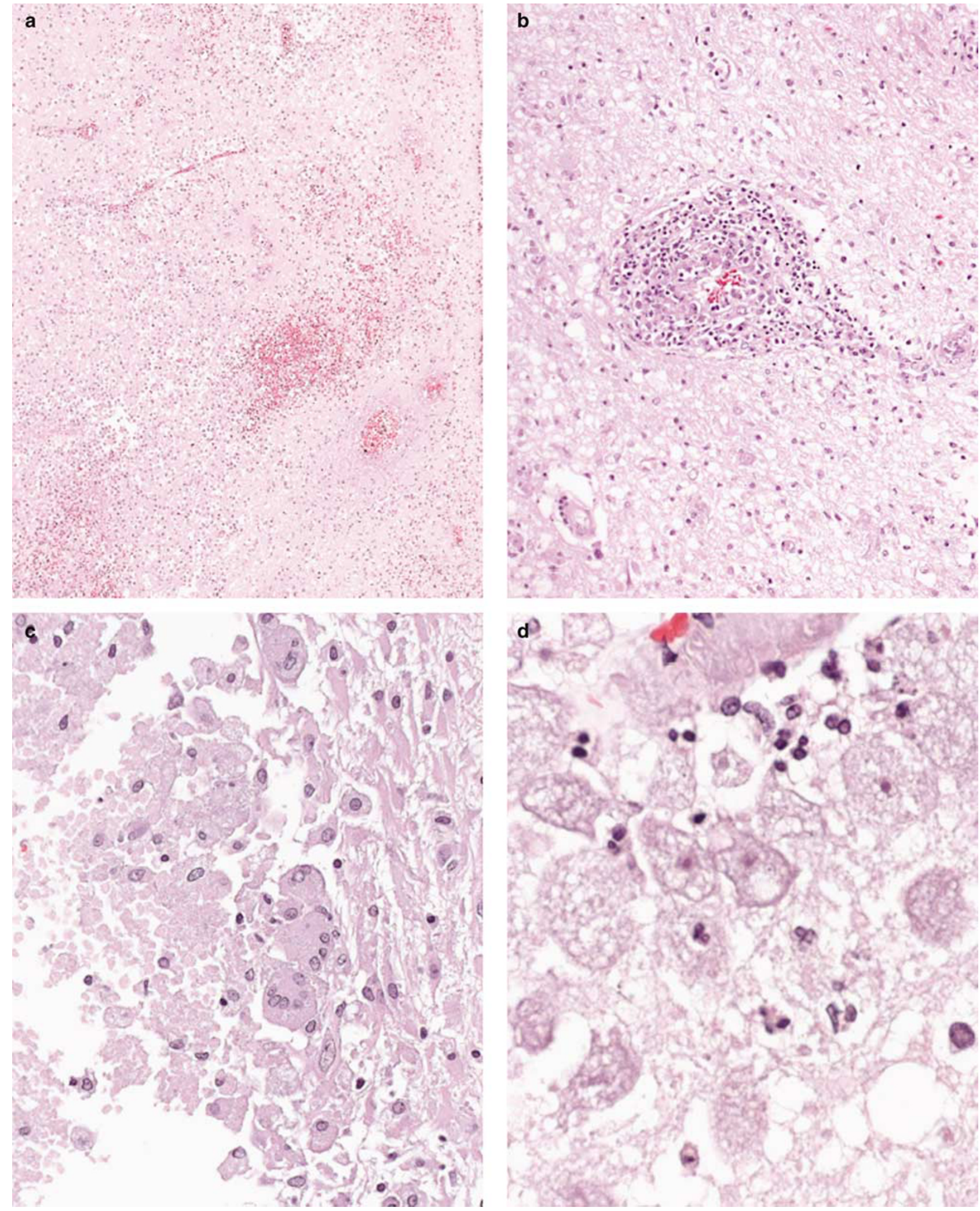

Figure 1 Histopathologic features of amebic central nervous system infections showing necrotizing encephalitis (a), angiitis (b), and granulomatous (c) and neutrophilic inflammation (d). Trophozoites are easily identifiable in a patient with Balamuthia infection (d). Hematoxylin and eosin stains of infection with Naegleria (a), Balamuthia (b and d), and Acanthamoeba (c). Original magnifications: (a) $\times 25,(\mathbf{b}) \times 50,(\mathbf{c}) \times 100$, (d) $\times 157$. 

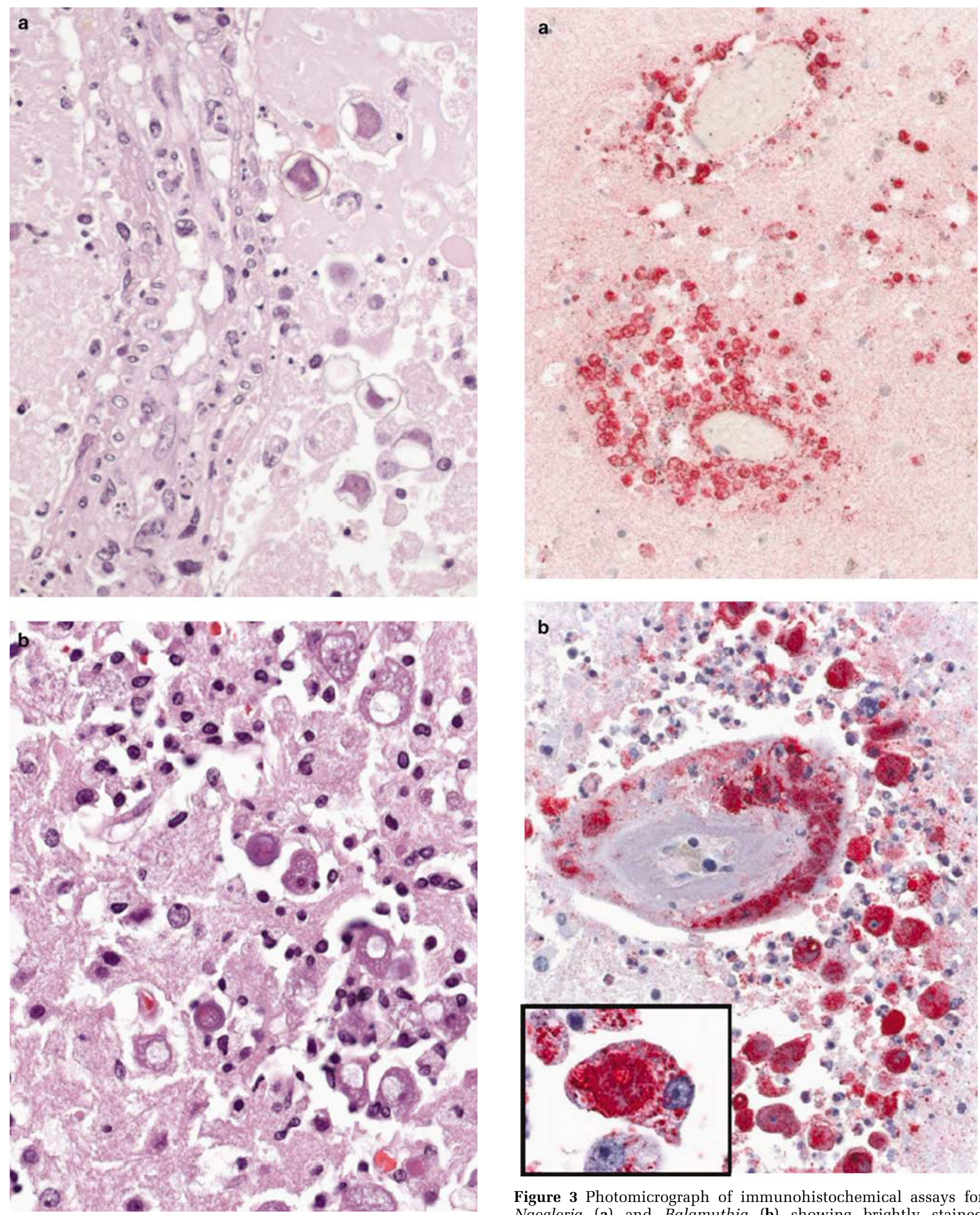

Figure 2 Morphologic characteristics of trophozoites and cysts present in cases with Acanthamoeba (a) and Balamuthia (b) infections. (Hematoxylin and eosin stains. Original magnifications: (a) $\times 100$, (b) $\times 157$.)

Figure 3 Photomicrograph of immunohistochemical assays for Naegleria (a) and Balamuthia (b) showing brightly stained trophozoites and granular antigens in the blood vessel walls and in macrophages (inset). (Immunohistochemistry using polyclonal antibodies against Naegleria and Balamuthia and naphthol fast red chromogenic substrate. Original magnifications: (a and b) $\times 100$, inset $\times 250$.) 
(Figure 3a) which had been difficult to identify with hematoxylin and eosin stains because their nuclei were not evident. Cyst walls were faintly stained with the immunohistochemical assays. Immunohistochemical assays demonstrated the presence of granular antigens prominently in vessel walls (Figure 3a and b) particularly for tissues of patients with Naegleria and Balamuthia infections. Granular antigen fragments were also observed inside macrophages (Figure $3 \mathrm{~b}$ inset) and endothelial cells. In one Balamuthia case, the Acanthamoeba antibody faintly stained the ameba, suggesting that there is some crossreactivity of the antibodies. Of the 42 cases tested with the ameba antibodies, 24 tested negative and included two patients with tuberculous meningitis, one with Streptococcus pneumoniae meningitis, one with rabies encephalitis, two with infarcts, and 18 with meningoencephalitis for which an infectious agent could not be determined.

\section{Discussion}

This comparative study of cases with central nervous system infections by free-living amebas showed that the current classification of primary amebic meningoencephalitis and granulomatous amebic encephalitis is not as clearcut as previously thought. In our study, N. fowleri infections were associated with abundant neutrophilic inflammation in the meninges similar to what has been described in primary amebic meningoencephalitis. Acanthamoeba infections showed predominantly granulomatous inflammation in the brain parenchyma corresponding to previously described granulomatous amebic encephalitis. ${ }^{1-4}$ However, the histopathology of Balamuthia infections showed a wider spectrum of the inflammatory reactions, from acute inflammation only to mixed inflammation with or without granulomas. The variations in the inflammatory reaction observed in cases with Balamuthia infections suggest there are cases with intermediate histopathologic features that do not conform to the currently established primary amebic meningoencephalitis and granulomatous amebic encephalitis nomenclature. Shirabe et $a l^{11}$ reported presence of neutrophilic inflammatory infiltrate with small granulomatous lesions in a 78-year old with $B$. mandrillaris meningoencephalitis and Sjogren's syndrome. They postulated that the intermediate pathologic features between primary amebic meningoencephalitis and granulomatous amebic encephalitis were a reflection of the altered immune status of the patient. Documentation of underlying health conditions that could alter the immune status in our patients with Balamuthia infections and intermediate histopathologic features was not available. Alternatively, the inflammatory reaction could reflect the duration of illness since Naegleria infections have a rapid clinical course and intense neutrophilic inflammation while Acanthamoeba infections have a protracted clinical course and granulomatous inflammation. ${ }^{2}$

We found meningoencephalitis in $88 \%$ of the cases regardless of the genera of ameba causing disease. It is postulated that $N$. fowleri enter through the olfactory mucosa causing acute meningitis, while Acanthamoeba and Balamuthia enter through lungs, skin, or mucous membranes and disseminate to the brain hematogenously causing parenchymal lesions such as abscesses or encephalitis. ${ }^{15,17}$ In our series, the meningeal inflammation appeared as a primary component of the disease process in patients with $N$. fowleri infections and prominent acute meningitis and in those with Balamuthia infections and granulomatous meningeal inflammation. The prominent meningeal inflammation in some patients with Balamuthia infection suggests possible entry of the ameba through olfactory mucosa. Since meningeal inflammation is a frequent occurrence for all free-living amebic infections of the central nervous system, the term meningoencephalitis describes better the histopathologic findings than the currently used classification of primary amebic meningoencephalitis and granulomatous amebic encephalitis.

For diagnosis of amebic infections, it is imperative to document the presence of amebic trophozoites or cysts. In the cerebrospinal fluid, $N$. fowleri trophozoites are frequently found while Acanthamoeba and Balamuthia are rarely observed. ${ }^{2}$ In our series, $50 \%$ of cases with Naegleria infections showed trophozoites in the meninges, 33\% with Acanthamoeba, and $18 \%$ with Balamuthia. Although trophozoites can be abundant and have been described as easily identifiable in tissue samples by using hematoxylin and eosin stains in the literature, in most of our cases the amebas were difficult to identify. The difficulty identifying amebas can relate to the number of parasites and the amount of inflammation and necrotic debris in the tissue section. ${ }^{20}$ In addition, differentiation of trophozoites from macrophages requires experience in recognizing the characteristic nuclear morphology of these parasites particularly in areas of intense inflammation and necrosis. An alternative to using specific immunohistochemical assays for amebas can be the use of immunohistochemical assays for macrophages such as CD68; as macrophages are highlighted, nuclear morphology can be studied in the nonstaining mononuclear cells which could correspond to amebas. Grocott's methenamine silver stains Acanthamoeba cysts and was useful in our cases to highlight them. ${ }^{23}$ However, the silver stains did not contribute further to what was already observed with hematoxylin and eosin stains where the characteristic cysts with wrinkled ectocyst walls were found in cases of Acanthamoeba infection. ${ }^{3}$ Cysts are not usually found in cases with $N$. fowleri infection because patients typically die before trophozoites can become encysted. In our series, ameba cysts were observed in three cases with 
Acanthamoeba infection and in three cases with Balamuthia. Interestingly, the cases with Balamuthia cysts had acute or mixed inflammatory pattern.

Fluorescent assays that use antibodies to detect amebic antigens have been used to highlight the presence of trophozoites. Colorimetric detection of antibodies in an immunohistochemical assay permitted the use of light microscopy and was helpful to better define the interaction of the free-living amebas in the pathological context. ${ }^{1,10-17}$ By using immunohistochemistry, trophozoites were highlighted in areas of necrosis or where there was intense inflammation and distinction between amebas and macrophages had been problematic with hematoxylin and eosin stains. It was also important in small biopsy specimens of patients who survived where the material examined was scant and consisted primarily of necrotic tissues. In addition, granular antigen staining, representing ameba fragments and antigens, was observed inside macrophages and endothelial cells, as well as extracellularly in vessel walls. Presence of Acanthamoeba antigens has been described in patients with amebic keratitis and has been postulated to account for persistent inflammatory reaction. ${ }^{24}$ Granular antigens in parasitic and bacterial diseases may explain continued host reaction to the microorganism and can be useful for diagnosis in the correct clinicoepidemiologic setting. ${ }^{21,22,25}$

Diagnostic techniques that do not rely on visualization of trophozoites, such as polymerase chain reaction, have been developed..$^{7,26-28}$ As molecular techniques are being applied more frequently for diagnosis of a variety of infectious agents, falsepositive reactions will occur. Thus, molecular results have to be correlated with epidemiologic, clinical, and pathologic features. For example, one of the patients evaluated with the anti-ameba antibodies had a false-positive polymerase chain reaction for Naegleria; however, light microscopy, immunohistochemistry, and fluorescent antibody assays failed to confirm the presence of ameba or granular antigens but demonstrated viral inclusions that were positive for rabies antigens by immunohistochemistry. ${ }^{29}$ This underscores the broad differential diagnosis of amebic meningoencephalitis, which includes bacteria (eg Mycobacterium tuberculosis, S. pneumoniae), neurotropic viruses, and noninfectious pathologies such as central nervous system infarcts.

In summary, the main histopathologic abnormality in cases with amebic infections of the central nervous system was meningoencephalitis. The spectrum of inflammation in cases of Balamuthia meningoencephalitis is broader than previously described. Immunohistochemical assays were useful to confirm the genus and highlight the ameba, particularly in necrotic areas. Presence of granular amebic antigens in vessel walls and endothelial cells may contribute to angiits.

\section{References}

1 Martinez A, Visvesvara G. Laboratory diagnosis of pathogenic free-living amoebas: Naegleria, Acanthamoeba, and Leptomyxid. Cli Lab Med 1991;11: 861-872.

2 Martinez A, Visvesvara G, Chandler F. Free-living Amebic Infections, 1st edn. Appleton and Lange: Stamford, CT, 1997

3 Ma P, Visvesvara G, Martinez A, et al. Naegleria and Acanthamoeba infections: review. Rev Infect Dis 1990;12:490-513.

4 Martinez A, Visvesvara G. Free-living, amphizoic and opportunistic amebas. Brain Pathol 1997;7:583-598.

5 Bloch K, Schuster F. Inability to make a premortem diagnosis of Acanthamoeba species infection in a patient with fatal granulomatous amebic encephalitis. J Clin Microbiol 2005;43:3003-3006.

6 Huang Z, Ferrante A, Carter R. Serum antibodies to Balamuthia mandrillaris, a free-living amoeba recently demonstrated to cause granulomatous amoebic encephalitis. J Infect Dis 1999;179:1305-1308.

7 Marciano-Cabral F, Cabral G. Acanthamoeba spp. as agents of disease in humans. Clin Microbiol Rev 2003;16:273-307.

8 Flores B, Garcia C, Stamm W, et al. Differentiation of Naegleria flowleri from Acanthamoeba species by using monoclonal antibodies and flow cytometry. J Clin Microbiol 1990;28:1999-2005.

9 Li Q, Yang X, Qian J. September 2004: a 6-year-old girl with headache and stiff neck. Brain Pathol 2005; 15:93-95.

10 Castellano-Sanchez A, Popp A, Nolte F, et al. Acanthamoeba castellani encephalitis following partially mismatched related donor peripheral stem cell transplantation. Transpl Infect Dis 2003;5: 191-194.

11 Shirabe T, Monobe Y, Visvesvara G. An autopsy case of amebic meningoencephalitis. The first Japanese case caused by Balamuthia mandrillaris. Neuropathology 2002;22:213-217.

12 Deol I, Robledo L, Meza A, et al. Encephalitis due to a free-living amoeba (Balamuthia mandrillaris): case report with literature review. Surg Neurol 2000;53: 611-616.

13 Martinez S, Gonzalez-Mediero G, Santiago P, et al. Granulomatous amebic encephalitis in a patient with AIDS: isolation of Acanthamoeba sp. group II from brain tissue and successful treatment with sulfadiazine and fluconazole. J Clin Microbiol 2000;38:3892-3895.

14 Denney C, Iragui V, Uber-Zak L, et al. Amebic meningoencephalitis caused by Balamuthia mandrillaris: case report and review. Clin Infect Dis 1997;25: 1354-1358.

15 Recavarren-Arce S, Velarde C, Gotuzzo E, et al. Amoeba angeitic lesions of the central nervous system in Balamuthia mandrilaris amoebiasis. Hum Pathol 1999;30:269-273.

16 Rideout B, Gardiner C, Stalis I, et al. Fatal infections with Balamuthia mandrillaris (a free-living amoeba) in gorillas and other Old World primates. Vet Pathol 1997;34:15-22.

17 Riestra-Castaneda J, Riestra-Castaneda R, GonzalezGarrido A, et al. Granulomatous amebic encephalitis due to Balamuthia mandrillaris (Leptomyxiidae): report of four cases from Mexico. Am J Trop Med Hyg 1997;56:603-607. 
18 Galarza M, Cuccia V, Sosa F, et al. Pediatric granulomatous cerebral amebiasis: a delayed diagnosis. Pediatr Neurol 2002;26:153-156.

19 Deetz T, Sawyer M, Billman G, et al. Successful treatment of Balamuthia amoebic encephalitis: presentation of 2 cases. Clin Infect Dis 2003;37: 1304-1312.

20 Cha J, Furie F, Kay J, et al. Case 39-2006: a 24-year-old woman with systemic lupus erythematosus, siezures, and right arm weakness. N Engl J Med 2006;355: 2678-2689.

21 Guraner J, Barteltt J, Zaki S, et al. Mouse model for Chagas disease: immunohistochemical distribution of different stages of Trypanosoma cruzi in tissues throughout infection. Am J Trop Med Hyg 2001;65: 152-158.

22 Guarner J, Greer P, Whitney A, et al. Pathogenesis and diagnosis of human meningococcal disease using immunohistochemical and PCR assays. Am J Clin Pathol 2004;122:754-764.

23 Tan B, Weldon-Linne C, Rhone D, et al. Acanthamoeba infection presenting as skin lesions in patients with the acquired immunodeficiency syndrome. Arch Pathol Lab Med 1993;117:1043-1046.

24 Yang Y, Matheson M, Dart J, et al. Persistence of acanthamoeba antigen following acanthamoeba keratitis. Br J Ophthalmol 2001;85:277-280.

25 Guarner J, Shieh W, Morgan J, et al. Leptospirosis mimicking acute cholecystitis among athletes participating in a triathlon. Hum Pathol 2001;32:750-752.

26 Tavares M, Costa JCd, Carpenter S, et al. Diagnosis of first case of Balamuthia amoebic encephalitis in Portugal by immunofluorescence and PCR. J Clin Microbiol 2006;44:2660-2663.

27 Booton G, Visvesvara G, Byers T, et al. Identification and distribution of Acanthamoeba species genotypes associated with nonkeratitis infections. J Clin Microbiol 2005;43:1689-1693.

28 Visvesvara G, De Jonckheere JF, Sriram R, et al. Isolation and moleculartyping of Naegleria fowleri from the brain of a cow that died of primary amebic meningoencephalitis. J Clin Microbiol 2005;43:4203-4204.

29 CDC. First human death associated with raccoon rabies-Virginia, 2003. MMWR 2003;52:1102-1103. 